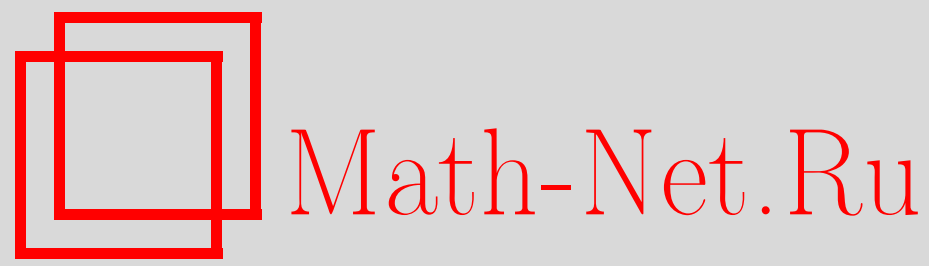

Н. А. Бокаев, Об абсолютной сходимости кратных рядов из коэффициентов Фурье по мультипликативным системам, Матем. заметки, 1996, том 60, выпуск 5, 765-767

DOI: https://doi.org/10.4213/mzm1888

Использование Общероссийского математического портала MathNet.Ru подразумевает, что вы прочитали и согласны с пользовательским соглашением

http://www . mathnet.ru/rus/agreement

Параметры загрузки:

IP : 52.6 .47 .48

26 апреля 2023 г., 11:07:25

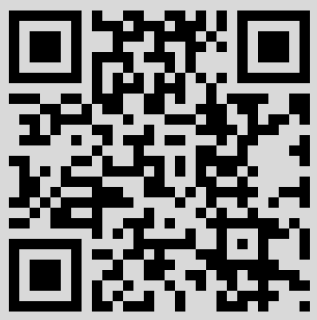




\section{ОБ АБСОЛЮТНОЙ СХОДИМОСТИ КРАТНЫХ РЯДОВ ИЗ КОЭФФИЦИЕНТОВ ФУРЬЕ ПО МУЛЬТИПЛИКАТИВНЫМ СИСТЕМАМ}

\section{H. А. Бокаев}

В настоящей работе в терминах полного группового модуля непрерьвности приводится достаточное условие сходимости рядов, составленных из коэффициентов Фурье функций многих переменных по мультипликативным системам, обобщающим систему Уолша. Показывается, что полученное условие неулучшаемо на классах функций $H_{p}^{\omega_{1}, \omega_{2}, \ldots, \omega_{n}}$.

Приведем необходимые определения и обозначения. Пусть $\left\{p_{k_{j}}^{(j)}\right\}_{k_{j}=1}^{\infty}(j=1,2$, $\ldots, n)$ - последовательности цельх чисел, $p_{k_{j}}^{(j)} \geqslant 2, j=1,2, \ldots, n, k_{j}=1,2, \ldots$, и пусть

$$
G^{(j)}=\left\{x=\left\{x_{k_{j}}^{(j)}\right\}_{k_{j}=1}^{\infty}, 0 \leqslant x_{k_{j}}^{(j)} \leqslant p_{k_{j}}^{(j)}-1\right\}, \quad j=1,2, \ldots, n,
$$

- соответствующие группы целочисленных последовательностей с групповой операцией $(\dot{+})$ покоординатного сложения по модулю $p_{k_{j}}^{(j)}, G^{n}=\prod_{j=1}^{n} G^{(j)}-$ прямое произведение групп $G^{(j)}$.

Множества $G_{\nu_{l}}^{(j)}=\left\{x=\left\{x_{k_{j}}^{(j)}\right\}_{k_{j}=1}^{\infty} \in G^{(j)}: x_{k_{j}}^{(j)}=0,0 \leqslant k_{j}<\nu_{l}\right\}$, $j=1,2, \ldots, n, \nu_{l}=1,2, \ldots, n$, являются подгруппами группы $G^{(j)}$ и система подгрупп $G\left(\nu_{l}\right)=\prod_{j=1}^{n} G_{\nu_{l}}^{(j)}\left(\nu_{l}=1,2, \ldots\right)$ группы $G^{n}$ задает систему окрестностей нуля в $G^{n}$. Относительно введенной операции сложения и топологии группа $G^{n}$ является компактной абелевой нульмерной группой (в одномерном случае см. [1]).

Положим $m_{0}^{(j)}=1, m_{r}^{(j)}=\prod_{k_{j}=1}^{r} p_{k_{j}}^{(j)}, j=1,2, \ldots, n, r=1,2, \ldots$.

Пусть на группе $G^{(j)}(1 \leqslant j \leqslant n)$ определена мультипликативная система Виленкина-Прайса (см. [1]):

$$
\begin{aligned}
\psi_{0}^{(j)} & =1, \quad x \in G^{(j)}, \\
\psi_{\nu_{j}}^{(j)}\left(x^{(j)}\right) & =\prod_{k_{j}=0}^{l\left(\nu_{j}\right)} \exp \frac{2 \pi i x_{k_{j}+1}^{(j)}}{p_{k_{j}+1}^{(j)}} \quad \text { при } \nu_{j}=\sum_{k_{j}=0}^{l\left(\nu_{j}\right)} \alpha_{k_{j}}^{\left(\nu_{j}\right)} m_{k_{j}}^{(j)},
\end{aligned}
$$

где $\alpha_{k_{j}}^{\left(\nu_{j}\right)}$ - целые числа, $0 \leqslant \alpha_{k_{j}}^{\left(\nu_{j}\right)}<p_{k_{j}+1}^{(j)}, x^{j}=\left\{x_{k_{j}}^{(j)}\right\}_{k_{j}=1}^{\infty} \in G^{j}$.

Система $\left\{\psi_{k_{j}}^{(j)}\left(x^{j}\right)\right\}_{k_{j}=0}^{\infty}(1 \leqslant j \leqslant n)$ является полной ортонормированной относительно меры Хаара $\mu_{j}$ на группе $G^{(j)}$ мультипликативной системой (см. [1]). При $p_{k_{j}}^{(j)}=2, k_{j}=1,2, \ldots$, система совпадает с системой Уолша в нумерации Пэли.

Работа выполнена при поддержке фонда INTAS, грант № 94-1044. 
Положим $\bar{k}=\left(k_{1}, k_{2}, \ldots, k_{n}\right), \bar{x}=\left(x_{1}, x_{2}, \ldots, x_{n}\right)$. Кратная система функций $\psi_{\bar{k}}(\bar{x})=\psi_{k_{1}, k_{2}, \ldots, k_{n}}\left(x^{(1)}, x^{(2)}, \ldots, x^{(n)}\right)=\psi_{k_{1}}\left(x^{(1)}\right) \psi_{k_{2}}\left(x^{(2)}\right) \cdots \psi_{k_{n}}\left(x^{(n)}\right)$ является полной ортонормированной относительно меры Хаара $\mu=\mu_{1} \times \mu_{2} \times \cdots$ $\times \mu_{n}$ на $G^{n}$ мультипликативной системой.

Пусть $f(\bar{x}) \in L_{p}\left(G^{n}\right), 1 \leqslant p<\infty$,

$$
\begin{aligned}
& \|f\|_{L_{p}\left(G^{n}\right)}=\int_{G^{n}}|f(\bar{x})|^{p} d \bar{\mu}(\bar{x}) \\
& \quad=\int_{G^{(1)}} \int_{G^{(2)}} \cdots \int_{G^{(n)}}\left|f\left(x^{(1)}, x^{(2)}, \ldots, x^{(n)}\right)\right| d \mu_{1} x^{(1)} d \mu_{2} x^{(2)} \cdots d \mu_{n} x^{(n)}
\end{aligned}
$$

и

$$
\sum_{k_{1}=0}^{\infty} \sum_{k_{2}=0}^{\infty} \ldots \sum_{k_{n}=0}^{\infty} \widehat{f}\left(k_{1}, k_{2}, \ldots, k_{n}\right) \psi_{k_{1}}\left(x^{(1)}\right) \psi_{k_{2}}\left(x^{(2)}\right) \cdots \psi_{k_{n}}\left(x^{(n)}\right)
$$

- ее ряд Фурье по системе $\psi_{k}(\bar{x})$, где

$$
\widehat{f}(\bar{k})=\int_{G^{n}} f(\bar{x}) \bar{\psi}_{\bar{k}}(\bar{x}) d \mu(\bar{x})
$$

- ее коэффиициенты Фурье.

Через

$$
\begin{aligned}
\omega_{p}(f, \bar{k})= & \omega_{p}\left(f ; k_{1}, k_{2}, \ldots, k_{n}\right) \\
= & \sup _{h^{(j)} \in G_{k_{j}}^{(j)}} \| f\left(x^{(1)} \dot{+} h^{(1)}, x^{(2)} \dot{+} h^{(2)}, \ldots, x^{(n)} \dot{+} h^{(n)}\right) \\
& -f\left(x^{(1)}, x^{(2)}, \ldots, x^{(n)}\right) \|_{L_{p}\left(G^{n}\right)}
\end{aligned}
$$

обозначим полный групповой модуль непрерьвности функций $f(\bar{x}) \in L_{p}\left(G^{n}\right)$, $1 \leqslant p<\infty$.

Справедливы следующие утверждения.

Tеорема 1. Пусть $1<p \leqslant 2,1 / p+1 / p^{\prime}=1,0<\beta \leqslant p^{\prime}, f(\bar{x}) \in L_{p}\left(G^{n}\right) u$

$$
\sum_{k_{1}=0}^{\infty} \sum_{k_{2}=0}^{\infty} \ldots \sum_{k_{n}=0}^{\infty}\left(\prod_{j=1}^{n} m_{k_{j}+1}^{(j)}\right)^{1-\beta / p^{\prime}} \omega_{p}^{\beta}\left(f ; k_{1}, k_{2}, \ldots, k_{n}\right)<\infty .
$$

Тогда сходится ряд

$$
\sum_{k_{1}=0}^{\infty} \sum_{k_{2}=0}^{\infty} \ldots \sum_{k_{n}=0}^{\infty}\left|\widehat{f}\left(k_{1}, k_{2}, \ldots, k_{n}\right)\right|^{\beta}<\infty
$$

Эта теорема в случае функций одной переменной доказана в работе [2].

В теореме образуюшие последовательности $\left\{p_{k_{j}}^{(j)}\right\}_{k_{j}=1}^{\infty}$ системы $\left\{\psi_{k_{j}}(x)\right\}_{k_{j}=1}^{\infty}$ $(1 \leqslant j \leqslant n)$ произвольны, в частности, они могут быть неограниченными в совокупности. В случае, когда $\sup _{1 \leqslant k_{j}<\infty} p_{k_{j}}^{(j)}=N_{j}<\infty, j=1,2, \ldots, n$, из теоремы 1 непосредственно вытекает 
СледствиЕ. Пусть $1<p \leqslant 2,1 / p+1 / p^{\prime}=1, f(\bar{x}) \in L_{p}\left(G^{n}\right)$,

$$
\sup _{1 \leqslant k_{j}<\infty} p_{k_{j}}^{(j)}=N_{j}<\infty
$$

$(j=1,2, \ldots, n) u$

$$
\sum_{k_{1}=0}^{\infty} \ldots \sum_{k_{n}=0}^{\infty}\left(\prod_{j=1}^{n} m_{k_{j}}^{(j)}\right)^{1-\beta / p^{\prime}} \omega_{p}^{\beta}\left(f ; k_{1}, k_{2}, \ldots, k_{n}\right)<\infty
$$

Тогда сходится ряд (2).

Это утверждение в одномерном случае доказано в [3] и является аналогом классической теоремы О. Саса о тригонометрических рядах. Оказьвается, что утверждение приведенного следствия имеет место только при условии ограниченности всех образующих последовательностей $\left\{p_{k_{j}}^{(j)}\right\}_{k_{j}=1}^{\infty}, j=1,2, \ldots, n$. При условии, когда хотя бы одна из последовательностей $\left\{p_{k_{j}}^{(j)}\right\}$ неограничена, сходимость ряда (3) может не обеспечить сходимость ряда из (2). А именно, справедлива

TEOPEMA 2. Пусть $1<p \leqslant 2,1 / p+1 / p^{\prime}=1,0<\beta \leqslant p^{\prime}, u$

$$
\sup _{1 \leqslant k_{j}^{\prime}<\infty} p_{k_{j^{\prime}}}^{\left(j^{\prime}\right)}=\infty
$$

при некотором $j^{\prime}\left(1 \leqslant j^{\prime} \leqslant n\right) u \sup _{1 \leqslant k_{j}<\infty} p_{k_{j}}^{(j)}=N_{j}<\infty$ nри всех $j \neq j^{\prime}$ $(1 \leqslant j \leqslant n)$. Тогда существует функиия $f(\bar{x}) \in L_{p}\left(G^{n}\right)$ такал, ито для нее ряд (3) сходится, однако, ряд (2) расходится.

Карагандинский государственный университет

Поступило им. Е. А. Букетова

29.04.96

\section{СПИСОК ЦИТИРОВАННОЙ ЛИТЕРАТУРЫ}

1. Агаев Г.Н., Виленкин Н.Я., Джафарли Г.М., Рубинштейн А.И. Мул типликативные системы функций и гармонический анализ на нульерных группах. Баку: Элм, 1981. 2. Quek T.S., Zap Y.H. // J. Math. Anal. Appl. 1980. V. 74. P. 1-14. 3. Onneweer C. W. // Duke Math. J. 1974. V. 41. P. 679-688. 4. ЖКантлесов ЖК. Ж. Коэффициенты Фурье и аппроксимативные свойства средних рядов Фурье по мультипликативной системе. Дисс. . . . к. ф.-м. н. Караганда: КарГУ, 1988. 